\title{
Pulsatile Tinnitus with a Dural Arterio-Venous Fistula Diagnosed by Computed Tomography-Angiography
}

\author{
Sujin Kim, Jaeyong Byun, Moonsuh Park and Sunkyu Lee \\ Department of Otorhinolaryngology-Head and Neck Surgery, Kyung Hee University Hospital at Gangdong, Seoul, Korea
}

$\begin{array}{ll}\text { Received } & \text { March 23, 2013 } \\ \text { Revised } & \text { September 11, } 2013 \\ \text { Accepted } & \text { October 18, 2013 }\end{array}$

Address for correspondence Sunkyu Lee, MD

Department of OtorhinolaryngologyHead and Neck Surgery,

Kyung Hee University Hospital at Gangdong, 892 Dongnam-ro,

Gangdong-gu, Seoul 134-727, Korea

Tel $+82-2-440-8160$

Fax $+82-2-440-8470$

E-mail pseudoi@hanmail.net
A 43 year-old female patient suffered the sudden onset of pulsatile tinnitus in the left ear 2 months ago. The tinnitus did not subside spontaneously and remained unchanged. The patient had no history of head trauma or surgery of the head and neck. The character of the tinnitus was pulsatile, and it was synchronous with the heart beat. Audiologic examinations were performed and all of the results were normal. Computed tomography with angiography was performed and evidence of an arterio-venous fistula (AVF) was found. 4-vessel angiography was performed to confirm the dural AVF between the external carotid artery and sigmoid sinus. Embolization of the feeder-vessels was done under a fluoroscope and $70 \%$ of the fistula flow was controlled after embolization and the tinnitus totally subsided during the embolization.

Korean J Audiol 2013;17:133-137

KEY WORDS: Pulsatile tinnitus · Arteriovenous fistula · Angiography · Embolization Therapeutic.

\section{Introduction}

Tinnitus is described as the perception of sound when there is no source of external sound. There are two types of tinnitus: somatoform tinnitus and sensorineural tinnitus. Somatoform tinnitus can be further divided into vascular tinnitus, myogenic tinnitus and patulous Eustachian tube syndrome. ${ }^{1)}$

Pulsatile tinnitus is a common clinical symptom, and it usually originates within a venous blood vessel. ${ }^{2)}$ Pulsatile tinnitus is known to result from non-laminar blood flow caused by increased blood flow or a reduced vascular cross sectional area. Pulsatile tinnitus can occur in conjunction with various diseases, including exudative otitis media, anemia, thyrotoxicosism, high jugular bulb, arteriovenous malformation, dural arterio-venous fistula (DAVF), skull base tumor and intracranial hypertension. ${ }^{3)}$ Among them, DAVF is reported as the most common cause of pulsatile tinnitus resulting from vascular lesions. ${ }^{4)}$

DAVF is defined as an abnormal connection between the

This is an Open Access article distributed under the terms of the Creative Commons Attribution Non-Commercial License (http://creativecommons. org/licenses/by-nc/3.0/) which permits unrestricted non-commercial use, distribution, and reproduction in any medium, provided the original work is properly cited. meningeal arteries and meningeal veins. DAVF commonly occurs in the cavernous sinus, lateral sinus and sigmoid sinus and is responsible for $10-15 \%$ of all intracranial vascular malformations while the incidence of DAVF is most common in the 40 to 60 years age group. ${ }^{5)}$ Although it is used as a standard diagnostic test, carotid angiography (CA) is not seen as a good screening tool because of its invasiveness and the associated risks. By contrast, computed tomography angiography (CTA) draws growing attention as a diagnostic tool for not only DAVF but also for other vascular lesions, and many studies support the efficacy of CTA. ${ }^{6)}$ We performed CTA to diagnose a patient suffering from pulsatile tinnitus, CA to make the final diagnosis and embolization for treatment of the patient. This report presents the results from our practices and review of the existing literature.

\section{Case Report}

A 43 year-old woman was admitted to hospital with headache of two months' duration and left-sided pulsatile tinnitus that occurred suddenly one month ago and had become severe. She complained of a constant rhythmic sound that beat in synchrony with her heartbeat. The sound became louder in her 
ear, making it difficult to sleep. On admission, the patient did not have any otologic symptoms such as otorrhea, dizziness and hearing loss except ear-fullness in the affected ear. She had no previous history of surgery, head and neck trauma, or systemic diseases. Based on the results of a questionnaire in which the answers were measured on a 10-point visual ana$\log$ scale (VAS), her intensity of tinnitus was 7, tinnitus-induced annoyance was 8 and the effects of the tinnitus on her life was 6 , showing a high level of discomfort. The tinnitus handicap inventory (THI) was also high, as much as 76 . The physical examination revealed no mass in the head and neck, and the otoscopy exhibited no abnormal characteristics of the tympanic membranes. No sound waves were detected by auscultation. The tinnitus sound became weaker when the patient turned her head to the left and when her neck was pressed with the hand. The result of the pure tone audiometric evaluation was $11 \mathrm{~dB}$ in the right ear and $18 \mathrm{~dB}$ in the left ear. The impedance audiometry produced a Type A curve for both ears. In the tinnitogram, the patient showed pulsatile tinnitus in the left ear. And the sound pressure was $24 \mathrm{~dB}$ over the frequency of $1000 \mathrm{~Hz}$. The results of the auditory brainstem response test and distortion product otoacoustic emission test were normal.

Based on the patient's condition and test results, pulsatile tinnitus was suspected, and CTA was performed as a screening test. In the CTA, a prominent vascular structure was observed along the left tentorial margin, and focal stenosis at the transverse-sigmoid junction was present. Distension of the left occipital artery was also observed (Fig. 1). The CTA findings suggested the possibility of a DAVF, and a 4-vessel CA was performed for the diagnosis.

Angiography of the left internal carotid artery revealed no abnormal findings in the arterial phase. However, the left external carotid angiogram revealed an enlarged occipital artery. Also, the blood drained to the left transverse sinus and the meningeal vein passing through the transcranial route, extending from the left occipital artery, middle meningeal artery, posterior auricular artery, and superficial temporal artery. The diagnosis of DAVF, fed by the left external carotid artery (ECA), and draining into the left transverse sinus, was therefore made (Fig. 2). Although excessive drainage into the cerebral cortex or brainstem was not observed, transarterial embolization was performed due to the severity of the tinnitus
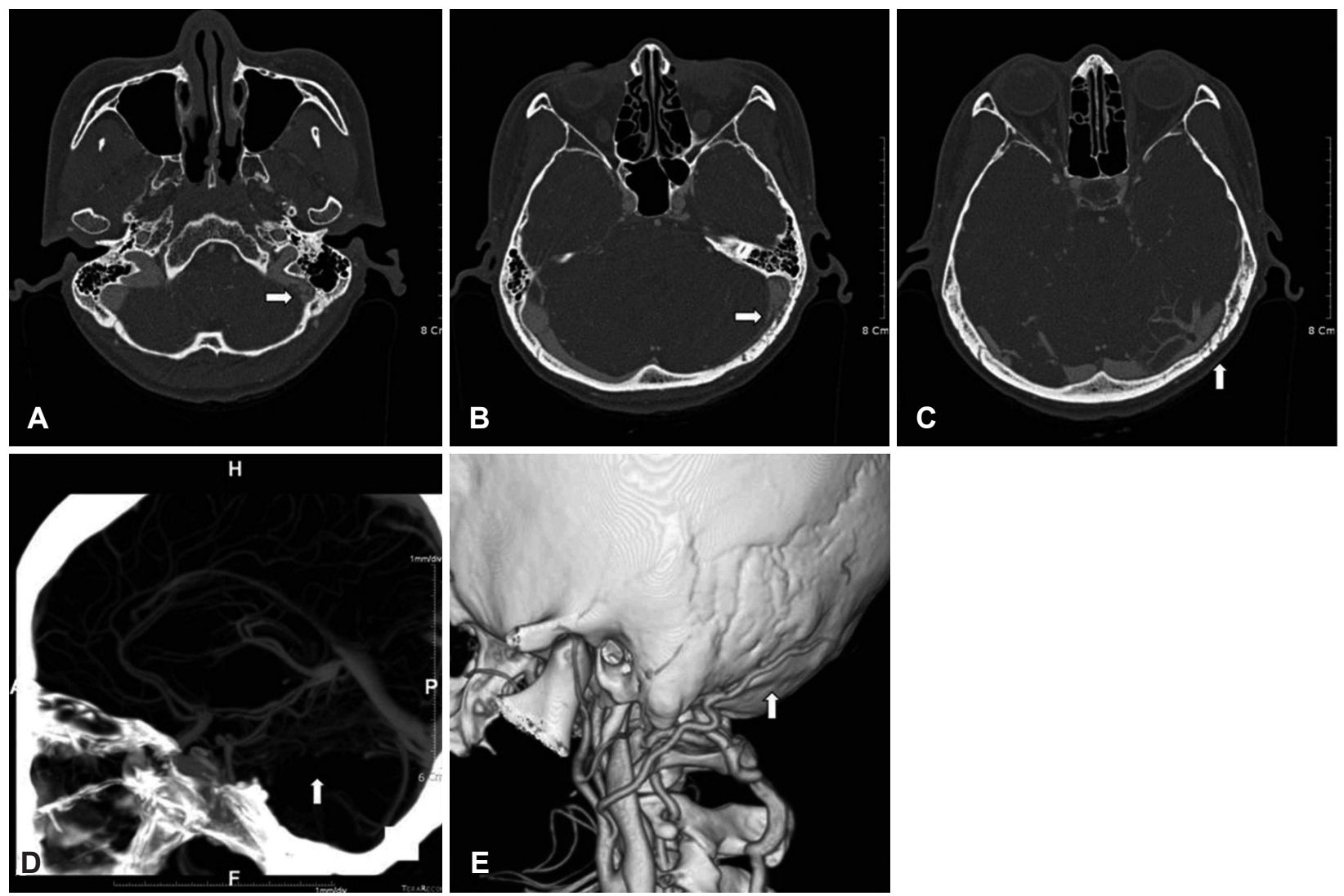

Fig. 1. Pre-embolization computed tomography angiography shows signs of dural arteriovenous fistula at transverse-sigmoid sinus and occipital artery. A: Shaggy appearance of sigmoid sinus (white arrow). B: Focal stenosis of left transverse-sigmoid junction (white arrow). C: Multiple bony channels at left occipital area (white arrow). D: Prominent vascular structures along left tentorial margin (white arrow). E: Dilated left occipital artery (white arrow). 


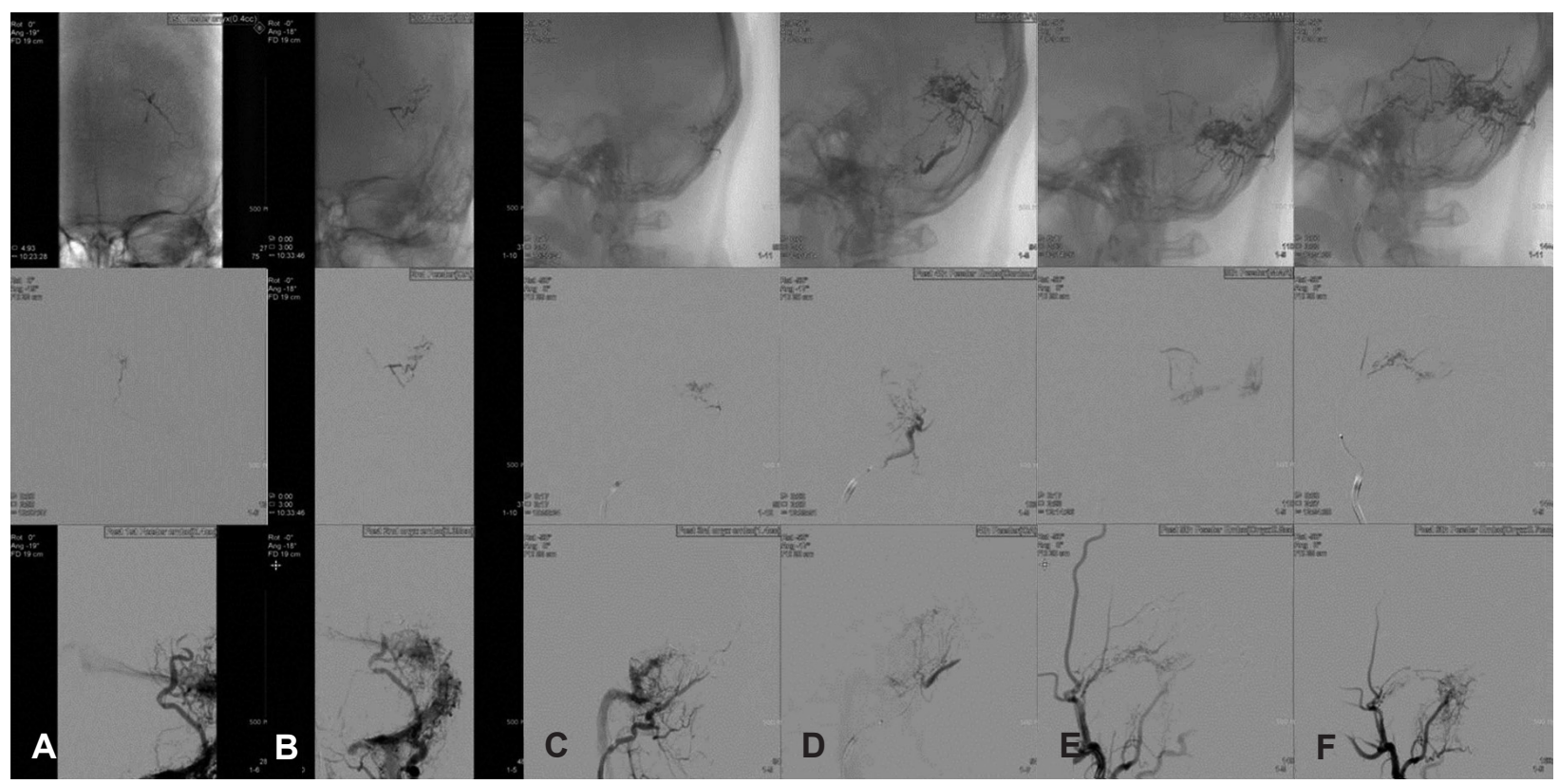

Fig. 2. Angiographic findings of arterial phase of left external carotid artery before and after embolization with N-butylcyanoacrylate. 1 st row is finding of pre-embolization, 2nd row intra-embolization, and 3rd row post-embolization. A: Coronal view of 1 st feeder from occipital artery. B: Coronal view of 2 nd feeder from occipital artery. C: Sagittal view of 3rd feeder from occipital artery. D: Sagittal view of 4th feeder from occipital artery. E: Sagittal view of 5th feeder from middle meningeal artery. F: 6th feeder from proximal portion of superficial temporal artery. Minimal blood flow to the sigmoid sinus through small collaterals of occipital artery is still visible in post-embolization.

symptoms and the high level of discomfort. First, a 6-Fr guiding catheter was placed through the left ECA to identify the enlarged occipital artery for catheterization. Embolizations with ethylene-vinyl alcohol copolymers (ONYX) were performed to embolize the four feeding arteries. Embolization of the middle meningeal artery was performed to embolize the fifth feeder, followed by transcranial arterial embolization in the proximal portion of the superficial temporal artery. A total of six feeding arteries were embolized. Post-embolization angiography revealed blood flow in fine arteries branching off from the posterior auricular artery and occipital artery. However, the feeders were too small and numerous to perform embolization on. During embolization, the pulsatile tinnitus disappeared, and no post-embolization complications were found. During the outpatient follow-up, the patient did not show tinnitus symptoms but claimed minor fullness in the ear. A THI questionnaire was conducted 3 months postoperatively, and the score for tinnitus-induced discomfort was 0 . However, the VAS score for annoyance associated with ear-fullness on the affected side was 2 .

\section{Discussion}

Pulsatile tinnitus is described as the perception of a rhythmic sound that beats in synchrony with the heartbeat. Pulsatile tinnitus can be subjective or objective. Possible mechanisms underlying pulsatile tinnitus include turbulences of blood flow, secondary symptoms of vascular stenosis and increased blood flow. While pulsatile tinnitus is caused by vascular or non-vascular abnormalities, the most common cause reported varies among studies. Sismanis ${ }^{3)}$ reported intracranial hypertension was responsible for $45 \%$ of all pulsatile tinnitus. Waldvogel, et al., ${ }^{4)}$ Dietz, et al. ${ }^{7)}$ claimed AVF as the most common cause. In general, vascular abnormalities are the main cause of pulsatile tinnitus. ${ }^{4)}$ Given that pulsatile tinnitus can be accompanied by serious complications, history-taking and physical examination must be performed. Based on the results of these examinations, determining whether radiological examination is necessary can be made. ${ }^{3)}$

DAVF is responsible for $2-20 \%$ of pulsatile tinnitus. ${ }^{4,8)}$ The mechanism underlying DAVF formation is not clear yet. However, researchers embrace the hypothesis claiming that DAVF may be caused by the combined effects of pathologically enlarged normal arterio-venous (AV) shunts (a normal AV shunt with a diameter of 50-90 $\mu \mathrm{m}$ exists in the dura mater) induced by sinus thrombosis and venous hypertension, and the recanalization process of the thrombosed sinus. ${ }^{9}$ DAVF develops most frequently near the transverse-sigmoid sinus, accounting for nearly $24.7-63 \%$ of all DAVFs. And the cavernous sinus is the second most frequent location for DAVF, accounting for $12-25.8 \%$ of all DAVFs.

If pulsatile tinnitus is not detected in the physical examination, there can be a dilemma in choosing the diagnostic tool because various differential diagnostic procedures are avail- 
able. Researchers can choose angiography, MRI/A, CT or CTA for the diagnosis. MRI/A is one of the most sensitive tests for diagnosis of DAVF, with a sensitivity of $50 \%$ to $100 \%$, and it is less invasive than angiography, making it popular as a diagnostic tool. ${ }^{10)}$ Angiography is an accurate test for the diagnosis of vascular lesions, allowing direct examination of the lesions. ${ }^{10)}$ However, it is invasive and poses a risk of complications. CTA showed a similar diagnostic accuracy with MRI/ $A$ in recent studies, and it has the advantages of simultaneous examination of the lesions of the temporal bone. CTA is more effective in diagnosing other causes of pulsatile tinnitus than DAVF. ${ }^{11)}$ Since CTA uses a lower dose of radiation compared to angiography, CTA is widely used for the early diagnosis of pulsatile tinnitus. ${ }^{11,12)}$

In CTA, DAVF is diagnosed by direct and indirect signs. CTA signs of DAVF include multiple arterial feeders, venous intimal thickening, stenosis and/or thrombosis, dilation of and reflux within venous efferents, numerous asymmetric and/or dilated arteries and venous collaterals, shaggy appearance of a dural venous sinus or the tentorium cerebelli, increased number and/or enlargement of cortical veins. ${ }^{6}$ )

In this report, DAVF was not detected by physical examination and audiometric evaluation. CTA was therefore performed as the initial diagnostic tool. DAVF was suspected based on a dilated occipital artery, shaggy appearance of the sigmoid sinus, focal stenosis of the transverse-sigmoid junction, and prominent vascular structure of the tentorial margin (Fig. 1). Subsequently, 4-vessel angiography was performed and DAVF between the left occipital artery and sigmoid sinus was diagnosed.

The treatment approach for DAVF depends on the clinical symptoms of the patient, the location of the lesion and direction of arterial drainage. Treatment is usually focused on complete obliteration but palliative treatment is also adopted for less severe cases. Treatment methods include conservative monitoring, endovascular embolization (transarterial and transvenous), surgical excision and radiation therapy. More than two methods are combined to maximize efficacy in most of the DAVF cases. But only the surgical procedure is effective for DAVF in the anterior cranial fossa and only endovascular embolization is effective for cavernous DAVF.

Embolization may be followed by complications such as a transient or permanent neurologic deficit resulting from ischemia and hemorrhage. The prevalence rate of complications is 3 to $11 \%{ }^{13)}$ Underlying factors associated with complications that have been cited are old age, the number of embolizations, the absence of pretreatment neurological deficits and periprocedural hemorrhage. ${ }^{14)}$ To discourage complications, softer flow-directed microcatheter has been recently devel- oped, and more liquid embolic agents such as n-butyl cyanoacrylate (NBCA) and ONYX are used. Liquid embolic agents allow nidal penetration during embolization, making extensive embolization to venous parts possible. ${ }^{15)}$

This report discusses transverse-sigmoid sinus DAVF in which the risks of complications such as reverse venous drainage or aneurysmal venous dilation were not found in our study. However, the patient had sleeping problems due to severe pulsatile tinnitus, so her DAVF needed to be treated. Transarterial embolization through the ECA was performed. To reduce the risk of complications, liquid ONYX was used as the embolic agent. A total of six embolizations were performed as six feeders were identified. Although post-embolization angiography revealed fistula flows fed by fine feeders, additional embolization was not performed because the pulsatile tinnitus disappeared.

Pulsatile tinnitus can occur with various systematic causes and intracranial diseases. First, history-taking needs to be performed and the symptoms of pulsatile tinnitus should be identified. And then the physical examination and radiological investigations, if necessary, can take place. CTA has emerged as a convenient and safe test for the examination of structural lesions and vascular lesions in the temporal bone. Its sensitivity and specificity are above $90 \%$. If a vascular lesion, especially DAVF is suspected, despite the negative findings on CTA, angiography should be performed because this procedure is useful for making decisions on therapeutic approaches and assessing the prognosis. Its invasiveness and high risks pose limitation. The use of CTA in the diagnosis of pulsatile tinnitus is however growing because of its convenience, safety and ability to detect various causes at the same time. We report a case of DAVF diagnosed with CTA and pulsatile tinnitus treated with subsequent therapeutic angiography and embolization.

\section{REFERENCES}

1) Park SN. Tinnitus: recent treatment. Res Vestib Sci 2009;8:108-16.

2) Madani G, Connor SE. Imaging in pulsatile tinnitus. Clin Radiol 2009;64:319-28.

3) Sismanis A. Pulsatile tinnitus. A 15-year experience. Am J Otol 1998; 19:472-7.

4) Waldvogel D, Mattle HP, Sturzenegger M, Schroth G. Pulsatile tinnitus--a review of 84 patients. J Neurol 1998;245:137-42.

5) Malek AM, Halbach VV, Higashida RT, Phatouros CC, Meyers PM, Dowd CF. Treatment of dural arteriovenous malformations and fistulas. Neurosurg Clin N Am 2000;11:147-66, ix.

6) Narvid J, Do HM, Blevins NH, Fischbein NJ. CT angiography as a screening tool for dural arteriovenous fistula in patients with pulsatile tinnitus: feasibility and test characteristics. AJNR Am J Neuroradiol 2011;32:446-53.

7) Dietz RR, Davis WL, Harnsberger HR, Jacobs JM, Blatter DD. MR imaging and MR angiography in the evaluation of pulsatile tinnitus. AJNR Am J Neuroradiol 1994;15:879-89.

8) Sonmez G, Basekim CC, Ozturk E, Gungor A, Kizilkaya E. Imaging 
of pulsatile tinnitus: a review of 74 patients. Clin Imaging 2007;31: 102-8.

9) Herman JM, Spetzler RF, Bederson JB, Kurbat JM, Zabramski JM. Genesis of a dural arteriovenous malformation in a rat model. J Neurosurg 1995;83:539-45.

10) Kim MS, Han DH, Kwon OK, Oh CW, Han MH. Clinical characteristics of dural arteriovenous fistula. J Clin Neurosci 2002;9:14755.

11) Christie A, Teasdale E. A comparative review of multidetector CT angiography and MRI in the diagnosis of jugular foramen lesions. Clin Radiol 2010;65:213-7.

12) Feygelman VM, Huda W, Peters KR. Effective dose equivalents to patients undergoing cerebral angiography. AJNR Am J Neuroradiol 1992;13:845-9.

13) Lv X, Wu Z, Jiang C, Li Y, Yang X, Zhang Y, et al. Complication risk of endovascular embolization for cerebral arteriovenous malformation. Eur J Radiol 2011;80:776-9.

14) Hartmann A, Pile-Spellman J, Stapf C, Sciacca RR, Faulstich A, Mohr JP, et al. Risk of endovascular treatment of brain arteriovenous malformations. Stroke 2002;33:1816-20.

15) Panagiotopoulos V, Gizewski E, Asgari S, Regel J, Forsting M, Wanke I. Embolization of intracranial arteriovenous malformations with ethylene-vinyl alcohol copolymer (Onyx). AJNR Am J Neuroradiol 2009;30:99-106. 